\title{
Laughter yoga reduces the cortisol response to acute stress in healthy individuals
}

\author{
Maria Meier ${ }^{\mathrm{a}}$, Lisa Wirz ${ }^{\mathrm{b}}$, Philip Dickinson ${ }^{\mathrm{c}}$ and Jens C. Pruessner ${ }^{\mathrm{a}, \mathrm{d}}$ \\ ${ }^{a}$ Department of Psychology, University of Constance, Constance, Germany; ${ }^{b}$ Department of Cognitive Neuroscience, Ruhr University Bochum, \\ Bochum, Germany; ${ }^{C}$ Centre de recherche de I'Institut universitaire de gériatrie de Montréal, Canada; ${ }^{d}$ Department of Psychiatry, Douglas \\ Mental Health Institute, McGill University, Montreal, Quebec, Canada
}

\begin{abstract}
Stress is one of the foremost contributors to the development of psychiatric diseases. Since the prevalence of stress-related complaints is increasing, we are in need for affordable and effective treatment alternatives. Laughter yoga (LY), a popular method encouraging participants to simulate laughter and participate in yogic breathing exercises, is hypothesized to buffer negative effects of stress. Although widely practiced, empirical evidence for beneficial effects of LY is scarce. We investigated the acute effects of a single 30-min LY session on the autonomic, endocrine and psychological response to a standardized psychosocial stressor. Thirty-five healthy subjects (51\% female) were randomly assigned to experience either a LY $(n=11)$, a relaxation breathing $(n=12)$ or a (non-intervention) control $(n=12)$ session prior to their exposure to the Trier Social Stress Test for Groups (TSST-G). Salivary cortisol, salivary alpha amylase, and subjective stress were assessed repeatedly throughout the experiment. We expected that LY and relaxation breathing group each show a downregulation of stress response indices compared to the control group. Further, we expected that LY has beneficial effects compared to relaxation breathing. The groups did not differ in salivary cortisol, alpha amylase or subjective stress reactivity during the 30-min intervention. However, in response to the TSST-G, the LY, but neither the relaxation breathing, nor the control condition, showed an attenuated cortisol stress response. These findings highlight the potential of LY to buffer the endocrine stress response. Therefore, LY could be used as a cheap and easily-to-implement add-on to more traditional stress interventions.
\end{abstract}

\section{LAY SUMMARY}

- In recent years, more and more people have reported to feel stressed. Although our body is well equipped to deal with acute stress, chronic stress can tire our system and contribute to illness in the long run. Therefore, we need affordable and effective measures to reduce stress. In this study we have investigated whether a single laughter yoga session can help us to deal with acute stress. Although laughter yoga did not change how stressful a situation was perceived, it reduced the amount of stress hormones that were released in response to the situation. As such, laughter yoga might be a cheap and easily-to-implement add-on to more traditional stress reduction interventions.

\section{KEYWORDS}

Laughter yoga; stress; cortisol; alpha amylase; Trier Social Stress Test for Groups; breathing

\section{Introduction}

The increasing prevalence of stress-related diseases and rising costs for health care systems (Kalia, 2002) create the need for affordable and effective treatments counteracting stress. In recent years, research has elucidated different interventions that are capable of attenuating the stress response to psychosocial stress, such as stress management training (Hammerfald et al., 2006), partner support in men (Kirschbaum et al., 1995), social interaction with humans (Ditzen et al., 2007), or dogs (Polheber \& Matchock, 2014) prior to the stressor. However, findings of the effects of such interventions on subjective and physiological stress responses have been equivocal. For example, interventions involving social support seem to decrease the physiological response to stress, but the situation itself is still perceived as being stressful (Ditzen et al., 2007). Social support by a partner can even increase the cortisol stress response in women (Kirschbaum et al., 1995). As an increasingly popular method in stress management mindfulness meditation training seems to attenuate subjective stress, and shows effects on circadian aspects of cortisol regulation in subjects with chronic illnesses (Matousek et al., 2011), but does not lead to decreased physiological stress responses (Creswell et al., 2014). Laughter, in contrast,seems to consist of social (communicative) and physiological (arousal) components, which may buffer negative effects of stress also acutely.

CONTACT Maria Meier maria.meier@uni-konstanz.de Department of Psychology, University of Constance, Constance, Germany

*Donders Institute for Brain, Cognition and Behaviour, Radboud University Medical Centre, Nijmegen, The Netherlands; Department for Cognitive Neuroscience, Radboud University Medical Centre, Nijmegen, The Netherlands. 
Laughter is a universal phenomenon characterized by strong exhalations and inhalations, with resulting increases in respiratory rate and breathing volume (Ruch \& Ekman, 2001). Gelotology - the research field investigating physiological, and psychological effects of laughter (Miller \& Fry, 2009) - is still in its infancy. An overarching idea is that laughter has positive effects on health (Martin, 2001; Savage et al., 2017; Yim, 2016), perhaps mediated by the capacity of laughter to modulate the relationship between stressful events and negative affect (Kuiper \& Martin, 1998). In an extreme example, individuals suffering from non-epileptic seizures often experience major stressors before, and feelings of calmness after seizures (Dickinson et al., 2011), showing the notable similarity in paroxysmal nature of pathological seizures and laughter.

In contrast to spontaneous laughter, simulated laughter is a self-induced form of laughter, which is independent of positive emotions or humor (Mora-Ripoll, 2011; Yim, 2016). The Indian physician Dr. Madan Kataria hypothesized similar positive physiological and psychological effects of spontaneous and simulated laughter (Fujisawa et al., 2018). Based on this assumption, he developed Laughter Yoga (LY), an exercise routine involving simulated laughter interspersed with deep yogic breathing (Bressington et al., 2018). Usually, LY is performed in groups to facilitate practice through the contagious character of laughter itself (Provine, 1992). If LY indeed excerpts stress buffering effects, it could offer a cost-efficient add-on to traditional stress-reducing approaches.

First studies supporting stress-reducing effects of laughter interventions, such as increases in self-efficacy, positive emotions, and reductions in stress and anxiety (Beckman et al., 2007; Chang et al., 2013; Farifteh et al., 2014; Heo et al., 2016; Weinberg et al., 2013), have been published recently. Some studies further showed modulating effects of LY on basal cortisol levels (Fujisawa et al., 2018; Tanaka et al., 2018). Previous studies have been criticized due to the lack of control groups and negligence of circadian variations of cortisol levels (Proyer et al., 2012), making it difficult to draw conclusions at this point (Proyer et al., 2012), and justifying further investigationson the effects of LY. The current experiment investigates whether LY has positive effects on physiological, and psychological responses to an acute stressor. Participants experienced either a LY, a (non-intervention) control, or a relaxation breathing session before experiencing a psychosocial stressor. We hypothesized that LY and relaxation breathing leads to significantly lower increases in cortisol, alpha amylase and subjective stress in response to the stressor when compared to the control group. Further we hypothesized that LY has additive benefits due to the theorized positive effects of laughter itself, thus, overall showing a stronger downregulation of stress indicators than the relaxation breathing group.

\section{Methods}

\section{Participants}

Thirty-five young healthy men and women between 18 and 34 years $\left(\right.$ mean $_{\text {age }}=23.77, S D_{\text {age }}=4.48 ; 51 \%$ female) from the Montréal area participated in the study. Through telephone screenings, the following exclusion criteria were applied: (1) a BMI smaller than 19 or larger than $27,(2)$ the consumption of more than 10 alcoholic drinks per week, (3) smoking, (4) traumatic brain injury followed by loss of consciousness, (5) drug abuse and addiction, (6) use of medications affecting the hypothalamic-pituitary-adrenal (HPA) axis (e.g. medication for asthma or arthritis), (7) suffering or having suffered from psychiatric disorders (according to DSM-V criteria), (8) a family history of psychiatric disorders, and (9) taking hormonal contraceptives (in female participants). All subjects gave written informed consent and received a compensation of CAN\$50. The study was approved by the Institutional Review Board of the Faculty of Medicine at McGill University and was performed in accordance with the Declaration of Helsinki.

\section{Study procedure}

Participants were invited to the experimental sessions in groups of four. In case of individual cancelations or unexpected absence of a participant, study assistants acted as dummy participants to keep the procedure standardized. Female participants were scheduled for participation during the luteal phase of their menstrual cycle to control for cyclic variations in hormonal levels and to get a physiological stress response comparable to that of male participants. For this purpose, the last two onsets of the menses, and the mean cycle length were recorded, while the luteal phase was defined as the last $45 \%$ of the cycle duration, and females were invited accordingly. Experimental sessions took around two and a half hours, and were scheduled to start at 12 p.m. to control for diurnal variations in the assessed biomarkers. The detailed study procedure is displayed in Figure 1.

After welcoming the participants and explaining the experimental procedure, participants gave written informed consent and filled in the Beck's Depression Inventory (BDI; Beck, Steer, \& Brown, 1996) and the State-Trait-AnxietyInventory (STAl; Spielberger, Sydeman, Owen, \& Marsh, 1999). This was followed by a 30-min intervention of either LY, relaxation breathing, or a (non-intervention) control session. Immediately after the intervention, psychosocial stress was induced by a modified version of the Trier Social Stress Test for Groups (TSST-G; von Dawans et al., 2011). The stressor was followed by a 40-min recovery phase, during which participants sat down, relaxed and were allowed to quietly read without further interacting with each other. Throughout the study procedure, participants were asked to provide saliva samples using Salivettes (Sarstedt, Quebec City, Canada) and

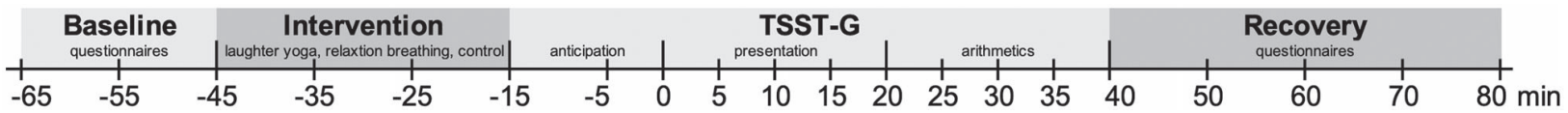

Figure 1. Overview of the study procedure. In total, 20 saliva samples and subjective stress ratings were taken. TSST-G: modified version of theTrier Social Stress Test for Groups. 
subjective stress ratings in 10-min intervals; during the TSST$G$ the measurements took place every $5 \mathrm{~min}$. At the end, participants were thanked, debriefed and compensated for their participation.

\section{Experimental groups}

Participants were randomly assigned to either LY $(n=11)$, relaxation breathing $(n=12)$ or (non-intervention) control group $(n=12)$. The LY sessions were led by an experienced instructor who gave a short introduction to LY and encouraged participants to laugh throughout the session, independent of whether laughter was forced or authentic. The LY instructor also proposed strategies to make it easier for participants to laugh, such as concentrating on another person's laughter that is experienced as enjoyable. Further, participants were motivated to interact and look at each other while laughing, to facilitate contagion (Provine, 1992). The experimenter and the LY instructor both participated in the session to motivate participants and facilitate interactions between the participants. During the first 20 participants performed different laughter exercises such as practicing, or imitating different types of laughter, and acting out different scenarios with each other, e.g. imagining seeing an old friend again. The goal was to make participants feel at ease, and to use exercises that might be helpful in inducing continuous laughter. In between exercises, participants took deep breaths to take a small break and to feel the relaxation following a laughter episode. In the last ten minutes, participants laid down on prepared mats on the floor, feet pointing toward each other in a circle. They placed their arms comfortably next to their bodies and spontaneously laughed in this position. The session ended with guided relaxation and deep breathing.

To control for changes in respiratory patterns during LY (Ruch \& Ekman, 2001), we included a relaxation breathing group in which different yogic breathing exercises were practiced in groups of four. Participants were standing in a circle and were instructed to maintain an upright and open body posture, use their diaphragms and lengthen respiration to reach the maximum of their total lung capacity and reduce residual lung volume as much as possible. The exercises were led by the experimenter who had previously been instructed by the LY teacher.

During the control session, subjects were invited in groups of four and could read magazines and books while sitting at individual tables, but were not allowed to interact with each other, or use cellphones or laptops.

\section{Trier social stress test for groups (TSST-G)}

Directly after the group interventions, participants were exposed to a modified version of the TSST-G (von Dawans et al., 2011). The TSST-G is a standardized modification of the Trier Social Stress Test (Kirschbaum et al., 1993), a widely used, and well-established stress protocol to induce psychosocial stress by combining high levels of uncontrollability and social-evaluative threat (Dickerson \& Kemeny, 2004). The
TSST-G has been shown to induce reliable increases in negative affect, as well as in autonomic nervous system, and HPA axis activity (e.g. Klaperski et al., 2013). We retrained the temporal specifications from the original TSST $(5 \mathrm{~min}$ speech and $5 \mathrm{~min}$ arithmetic problem solving) to create an overall longer stressful period, and maximize the stimulation of the HPA axis. As two of our experimental manipulations (laughter yoga and breathing) included an intervention aiming at reducing the stress response, we chose this procedure to avoid a bottom effect, where we would not be able to observe group differences because of insufficient stress responses.

After a 15-min preparation period, participants performed a mock job interview in front of a reserved, and non-reinforcing panel (one male, one female panelist) that evaluated the participants performance on two tasks. Firstly, participants gave a free speech about why he or she is the ideal candidate for a job tailored to his or her interests $(5 \mathrm{~min}$ for each participant). Secondly, participants performed a difficult mental arithmetic task by counting backwards from 2043 in steps of 17 ( $5 \mathrm{~min}$ for each participant). During both tasks, participants were videotaped and separated by partitions to prevent social interactions. The order in which they completed the tasks was randomly assigned.

\section{Physiological measures}

Throughout the experiment, 20 salivary samples were obtained at the timepoints depicted in Figure 1 using Salivette ${ }^{\circledR}$ devices (Sarstedt Inc., Quebec City, Canada) for subsequent analysis of alpha amylase and cortisol levels as markers of the sympathetic adrenal medullary (SAM) and HPA axis activity, respectively. Samples were stored at $-20^{\circ} \mathrm{C}$ until analysis using the enzymatic kinetic method for alphaamylase and a time-resolved fluorescence immunoassay for cortisol, both with proven reliability and validity.

\section{Subjective stress ratings}

Subjective stress levels were assessed throughout the experiment by a visual analogue scale (VAS) ranging from 0 ("not stressed") to 100 ("extremely stressed"). The VAS was filled in by the participants concordantly to providing the saliva samples.

\section{Statistical analysis}

All analyses were computed in $R$ version 3.5.3 ( $\mathrm{R}$ Core Team, 2019) using RStudio version 1.1.463 (RStudio Team, 2016) and the packages car (Fox \& Weisberg, 2011), multcomp (Hothorn et al., 2008), and effects (Fox \& Weisberg, 2019). Figures were created with ggplot2 (Wickham, 2016).

A total of 44 cortisol values (6.29\%) were missing due to insufficient amounts of saliva provided in the samples. Missing values were imputed either by the mean of the experimental condition if the value was missing at the first, group peak or last sample, or linearly in all remaining samples. We winsorized the existing cortisol data by replacing 
values that exceeded the mean by more than $3 S D$ with $3 S D$. This applied for $2.14 \%$ of the values.

The same procedure was applied for alpha amylase (with a total of $1.86 \%$ winsorized values and 56 missing values $[8 \%]$ ), and the subjective stress ratings (with a total of $.43 \%$ winsorized values and 74 missing values [10.57\%]). One-way ANOVAs (type II) with experimental condition (LY, relaxation breathing, control) as independent variable were performed to assure equal distribution of age (in years), BDI scores, STAI trait scores, cortisol baseline levels (first saliva sample) and alpha amylase baseline levels (first saliva sample) across the three groups. Variables that were not equally distributed across the groups were considered as confounds and were controlled for in subsequent analyses.

Physiological reactivity during the intervention (index $i$ for intervention) was measured by calculating the area under the individual response curves with respect to the increase (AUCi; Pruessner et al., 2003) during the time period of the intervention (from minute -45 to minute -15 ) for the alpha amylase $\left(A \cup\left(a_{i}\right)\right.$, and cortisol levels $\left(A \cup C\right.$ cort $\left._{\mathrm{i}}\right)$. The AUCi is used to incorporate multiple measurement points with varying time distance between the measures by estimating the integral of the curve in respect to the first value. As such, the AUCi is considered as the total increase in the released hormone, or enzyme during the given time period. The same procedure was applied for the subjective stress ratings to estimate the increase in subjective stress (AUCvas $)_{\mathrm{i}}$ ).

Accordingly, the physiological reactivity to the stressor (index $\mathrm{s}$ for stress) was assessed by calculating the AUCi during the time period of the TSST-G and the recovery phase (from minute -15 to minute +80 ) for the alpha amylase $\left(A \cup C a a_{s}\right)$, cortisol $\left(A \cup C C t_{s}\right)$, and the subjective stress levels (AUCvas $)_{\text {s }}$.

Two-way Analysis of Variances (ANOVAs) (type I) with different physiological and psychological measures as dependent variable (AUCsaa, AUCcort and AUCvas for the reactivity to the intervention and the stressor, respectively) and experimental condition ( $L Y$, relaxation breathing, control) and the confounded variables as independent variables were performed to test the interaction between the experimental condition and the confound variables. Whenever the interaction term did not reach statistical significance, a two-way ANOVA (type II) was used to estimate the main effect of experimental condition (LY, relaxation breathing, control) more robustly while controlling for the confounds, without taking the interaction into account. Additionally, post-hoc Tukey comparisons were performed to evaluate differences between the experimental conditions. Partial eta squared $\left(\eta_{p}{ }^{2}\right)$ was calculated as an indicator of effect size (Cohen, 1973; Lakens, 2013). In multifactorial designs, partial eta squared estimates the proportion of variance explained by one factor after deducting the proportion of variance that is explained by other factors in the analysis (Levine \& Hullett, 2002). In accordance with the benchmarks from Cohen, values of 0.01 account for a small effect, values of 0.06 account for a medium effect, and values of 0.14 account for a large effect (Cohen, 1988). The level of significance was set at $\alpha=.05$.

\section{Results}

Participants in the groups did not differ with respect to their $\operatorname{BDI}\left(F_{(2,32)}=1.64, p=.210, \eta_{p}{ }^{2}=0.09\right)$ or STAl trait scores $\left(F_{(2,}\right.$ $\left.\left.{ }_{32}\right)=0.68, p=.515, \eta_{p}{ }^{2}=0.04\right)$ and their basal cortisol (first saliva sample; $\left.F_{(2,32)}=1.58, p=.222, \eta_{p}{ }^{2}=0.09\right)$ or alpha amylase levels (first saliva sample; $\left.F_{(2,}, 32\right)=0.39, p=.681, \eta_{p}{ }^{2}=0.02$ ). However, participants in the LY group were significantly younger than those in the control group $\left(F_{(2,32)}=4.03, p=.027\right.$, $\left.\eta_{p}{ }^{2}=0.20\right)$, which is why age was considered and entered as confound in the subsequent analyses (see Table 1).

\section{Response to the intervention}

Analyzing the cortisol release during the intervention, there was no significant interaction between the experimental condition and age (ANOVA type $\mathrm{l} ; F_{(2,29)}=0.30, p=.745$, $\left.\eta_{p}{ }^{2}=0.23\right)$. The subsequent ANOVA showed that the confounded variable age was not significantly related to the cortisol release during the intervention (ANOVA type $\|_{\text {; }} F_{(1}$, 31) $\left.=0.30, p=.847, \eta_{p}{ }^{2}<0.01\right)$. In addition, the experimental conditions did not show a significant difference in their cortisol response to the intervention, while controlling for the effect of age $\left(F_{(2,31)}=0.30, p=.847, \eta_{p}{ }^{2}=0.02\right)$.

There was no significant interaction between the experimental condition and age predicting the increase in alpha amylase in response to the intervention (ANOVA type I; $\left.F_{(2,29)}=0.80, p=.457, \eta_{p}{ }^{2}=0.05\right)$. Similar to the cortisol reaction in response to the intervention, the following ANOVA showed neither an effect of age (ANOVA type $I$; $F_{(1,}$ $\left.31)=0.13, p=.721, \eta_{p}{ }^{2}<0.01\right)$, nor an effect of experimental condition $\left(F_{(2,31)}=0.31, p=.735, \eta_{p}{ }^{2}=0.02\right)$ in alpha amylase responses to the intervention.

The subjective stress in response to the intervention was not predicted by an interaction between the experimental condition and age (ANOVA type $\mathrm{l} ; F_{(2,29)}=0.72, p=.495$, $\eta_{p}{ }^{2}=0.05$ ). Neither age (ANOVA type $\| ; F_{(1,31)}=0.06$, $\left.p=.805, \eta_{p}^{2}<0.01\right)$, nor the experimental condition $\left(F_{(2}\right.$, 31) $\left.=0.01, p=.986, \eta_{p}{ }^{2}<0.01\right)$ had a significant effect on the subjective stress increases during the intervention.

\section{Response to the stressor}

After controlling for the effect of age, the ANOVA comparing the cortisol stress response between the groups showed a significant main effect of experimental condition (ANOVA type II; $\left.F_{(2,31)}=4.10, p=.026, \eta_{p}{ }^{2}=0.21\right)$. Tukey post-hoc $t$ tests with the confound adjusted means revealed significantly lower cortisol levels in the LY group $\left(\right.$ mean $_{\mathrm{adj}}=-3.44$, $\mathrm{se}_{\text {adj }}=99.64$ ) compared to the control group (meanadj $=365.98, \quad \mathrm{se}_{\mathrm{adj}}=100.11 ; \quad$ difference $=-369.42, \quad t=-2.51$, $p=.045$ ). The relaxation breathing group $\left(\right.$ mean $_{\mathrm{adj}}=324.95$, $\left.\mathrm{se}_{\mathrm{adj}}=94.22\right)$ neither differed significantly from the LY group (difference $=-328.39, t=-2.44, p=.052$ ), nor from the control group (difference $=41.03, t=0.29, p=.955$; see Figure 2 ). The ANOVA indicated that the confounded variable age was not significantly related to the cortisol increase $\left(F_{(1,31)}=1.33\right.$, $\left.p=.258, \eta_{p}{ }^{2}=0.04\right)$. There was no significant interaction between the experimental condition and age when analyzing 
Table 1. Descriptive statistics of the experimental condition.

\begin{tabular}{|c|c|c|c|c|}
\hline Variable & Laughter Yoga $(n=10)$ & Relaxation breathing $(n=12)$ & Control group $(n=12)$ & $p$ \\
\hline Age (years) & $22.09 \pm 1.89^{*}$ & $22.58 \pm 4.08$ & $26.50 \pm 5.45^{*}$ & $\bar{p}=.027$ \\
\hline BDI (sum score) ${ }^{\mathrm{a}}$ & $4.45 \pm 4.50$ & $7.67 \pm 4.87$ & $8.67 \pm 7.45$ & $p=.21$ \\
\hline STAI trait ${ }^{\mathrm{b}}$ & $36.09 \pm 10.71$ & $37.25 \pm 6.96$ & $40.50 \pm 10.44$ & $p=.515$ \\
\hline Cortisol baseline & $6.37 \pm 3.63$ & $4.54 \pm 1.62$ & $5.98 \pm 2.34$ & $p=.222$ \\
\hline Alpha amylase baseline & $84.79 \pm 71.32$ & $85.38 \pm 76.93$ & $108.68 \pm 76.62$ & $p=.681$ \\
\hline
\end{tabular}

Note. Data is expressed as mean $\pm S D$. One-way Analysis of Variances by experimental condition were calculated to test whether experimental groups differed in respect to the listed variables.

* Indicates a significant difference in means between the groups as revealed in pairwise $t$-tests with pooled SD and holm adjustment.

aDDI: Becks Depression Inventory II.

${ }^{b}$ STAI: State Trait Anxiety Inventory.

(A)

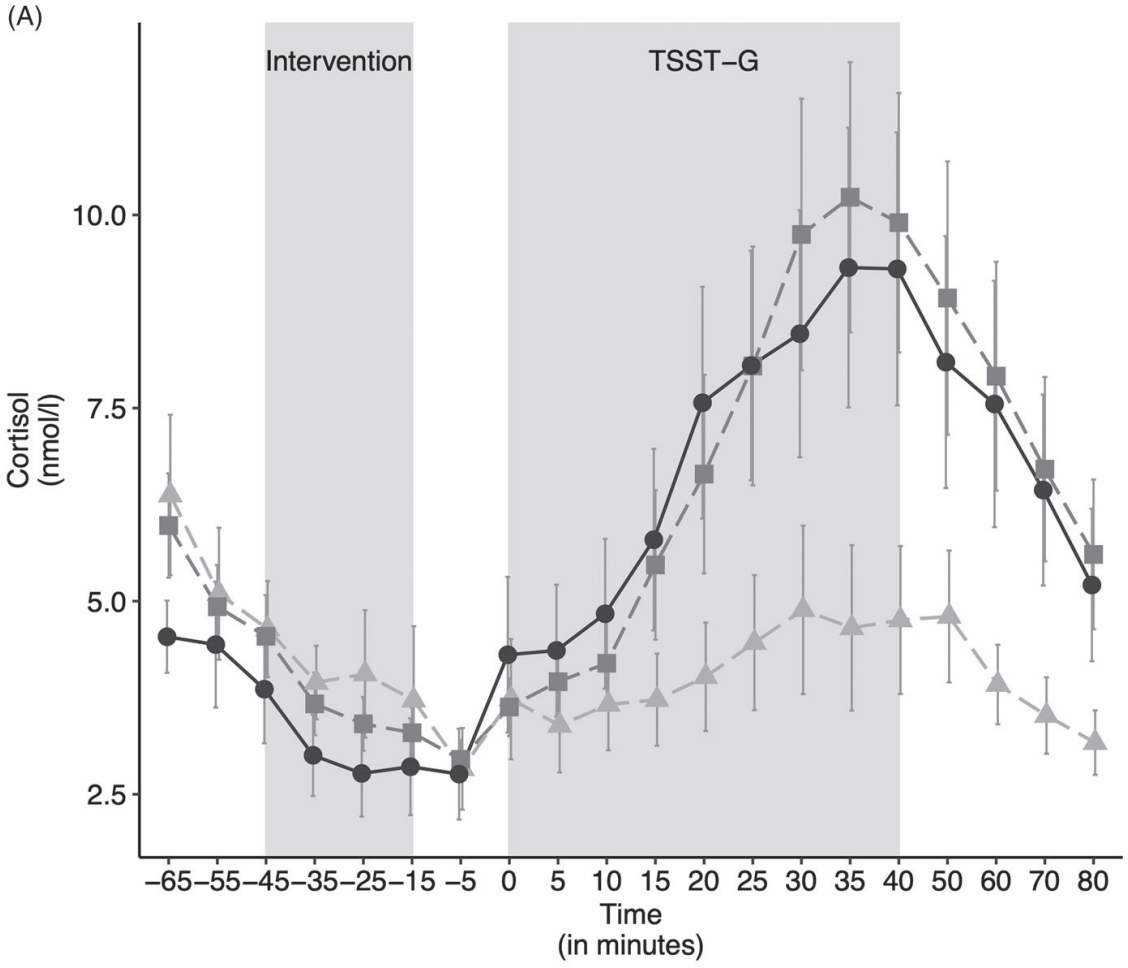

(B)

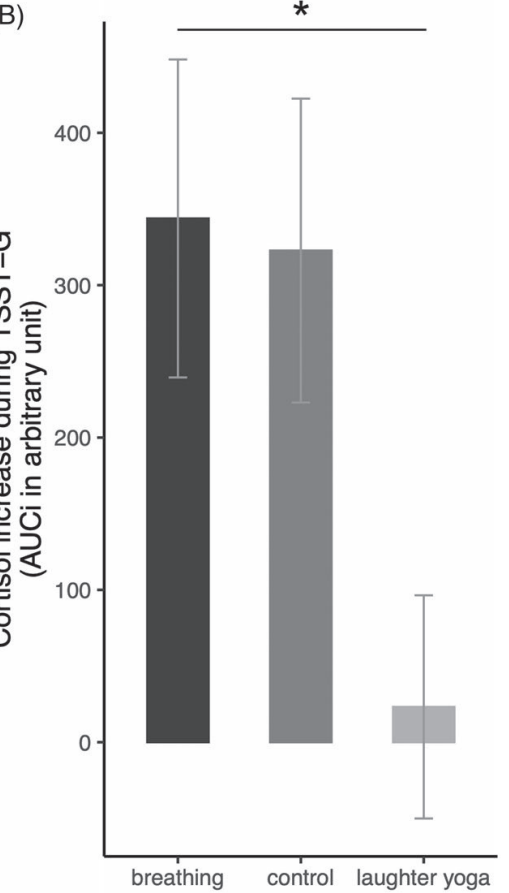

Experimental Group

Experimental Group —— Relaxation Breathing - - Control - - Laughter Yoga

Figure 2. (A) Salivary cortisol levels over the course of the experiment in the three experimental groups. (B) The cortisol increase in response to the stressor, measured by the area under the curve with respect to the increase (AUCi), by experimental group. The cortisol increase in response to the stressor differed significantly between the groups. The cortisol increase in the laughter yoga condition was significantly smaller compared to the control group. Values are shown as mean \pm SD. TSST-G: modified version of the Trier Social Stress Test for Groups.

the cortisol increase in response to the stressor (ANOVA type l; $\left.F_{(2,29)}=2.00, p=.154, \eta_{p}^{2}=0.12\right)$.

With respect to alpha amylase levels in response to stress, there was no significant interaction between the experimental condition and age (ANOVA type I; $F_{(2,29)}=0.15, p=.863$, $\eta_{p}{ }^{2}=0.05$ ). Neither age (ANOVA type $\|_{;} ; F_{(1,31)}=0.630$, $\left.p=.433, \eta_{p}{ }^{2}=0.02\right)$, nor our experimental condition had a significant effect on alpha amylase levels $\left(F_{(2,31)}=1.00\right.$, $p=.380, \eta_{p}{ }^{2}=0.06$; see Figure 3 ).

There was no significant interaction between the experimental condition and age when predicting the subjective stress increase in response to stress (ANOVA type $\mathrm{I} ; F_{(2,29)}=2.13$, $p=.137, \eta_{p}{ }^{2}=0.13$ ). Also, neither age (ANOVA type $\|_{;} F_{(1,}$ 31) $\left.=0.09, p=.761, \eta_{p}{ }^{2}<0.01\right)$, nor our experimental condition alone showed a significant effect on subjective stress increase $\left(F_{(2,31)}=0.20, p=.818, \eta_{p}{ }^{2}=0.01\right.$; see Figure 4$)$.

\section{Discussion}

This study investigated the effects of laughter yoga (LY) on the acute subjective, autonomic and endocrine stress response in a controlled experiment by comparing LY to both, a relaxation breathing and a (non-intervention) control group. Using the area under the curve with respect to the increase (AUCi), we found that the cortisol increase in response to a modified version of the Trier Social Stress Test for Groups (TSST-G; von Dawans et al., 2011) was significantly smaller in the LY condition compared to the control group, with a trend in the same direction in the comparison between the LY and the relaxation breathing group. This effect could neither be explained by baseline differences in cortisol levels at the onset of the experiment between the groups, nor by differences in the psychological, autonomic, 
(A)

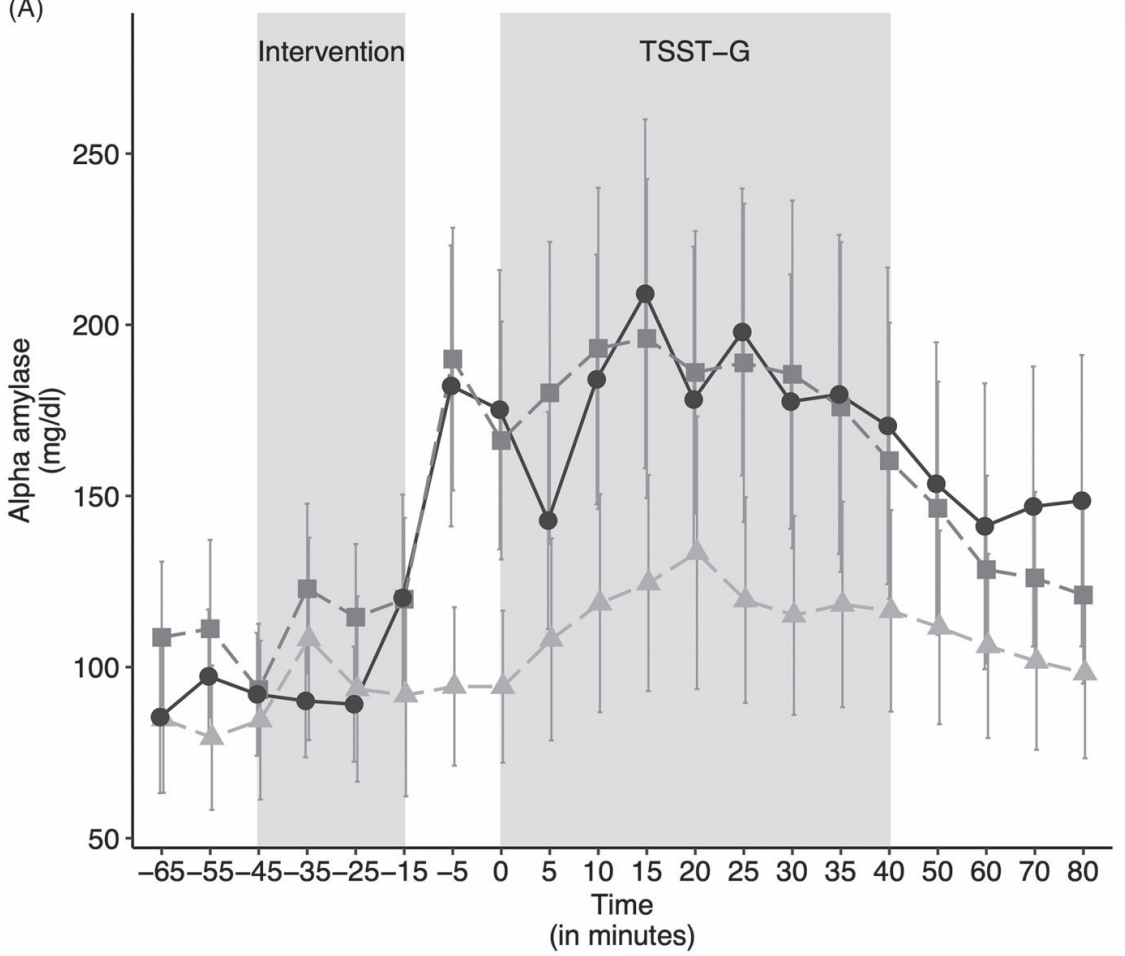

(B)

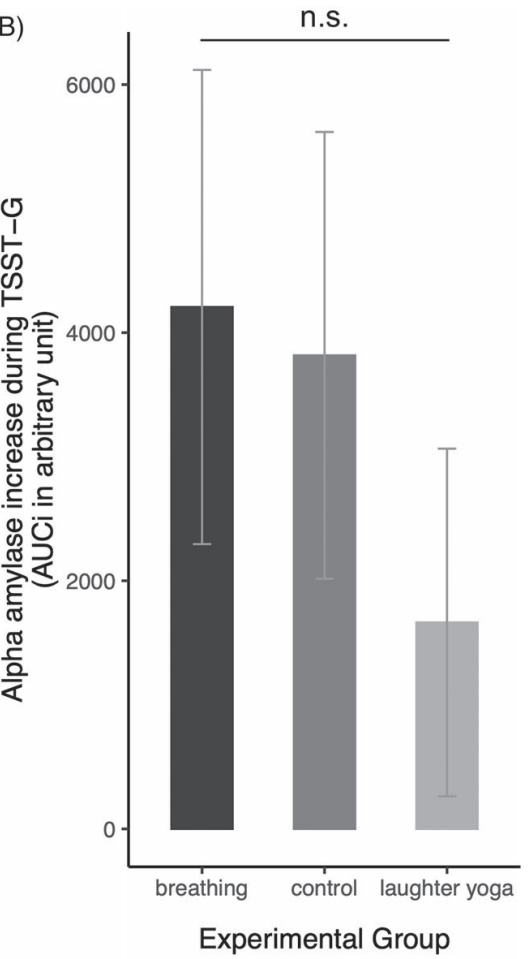

Experimental Group - - Relaxation Breathing $-\frac{-}{2}-$ Control $-1-$ Laughter Yoga

Figure 3. (A) Salivary alpha amylase levels over the course of the experiment in the three experimental groups. (B) The alpha amylase increase in response to the stressor, measured by the area under the curve with respect to the increase (AUCi), by experimental group. The alpha amylase increase in response to the stressor did not differ significantly between the groups. Values are shown as mean \pm SD. TSST-G: modified version of the Trier Social Stress Test for Groups.

(A)

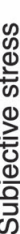

(A)

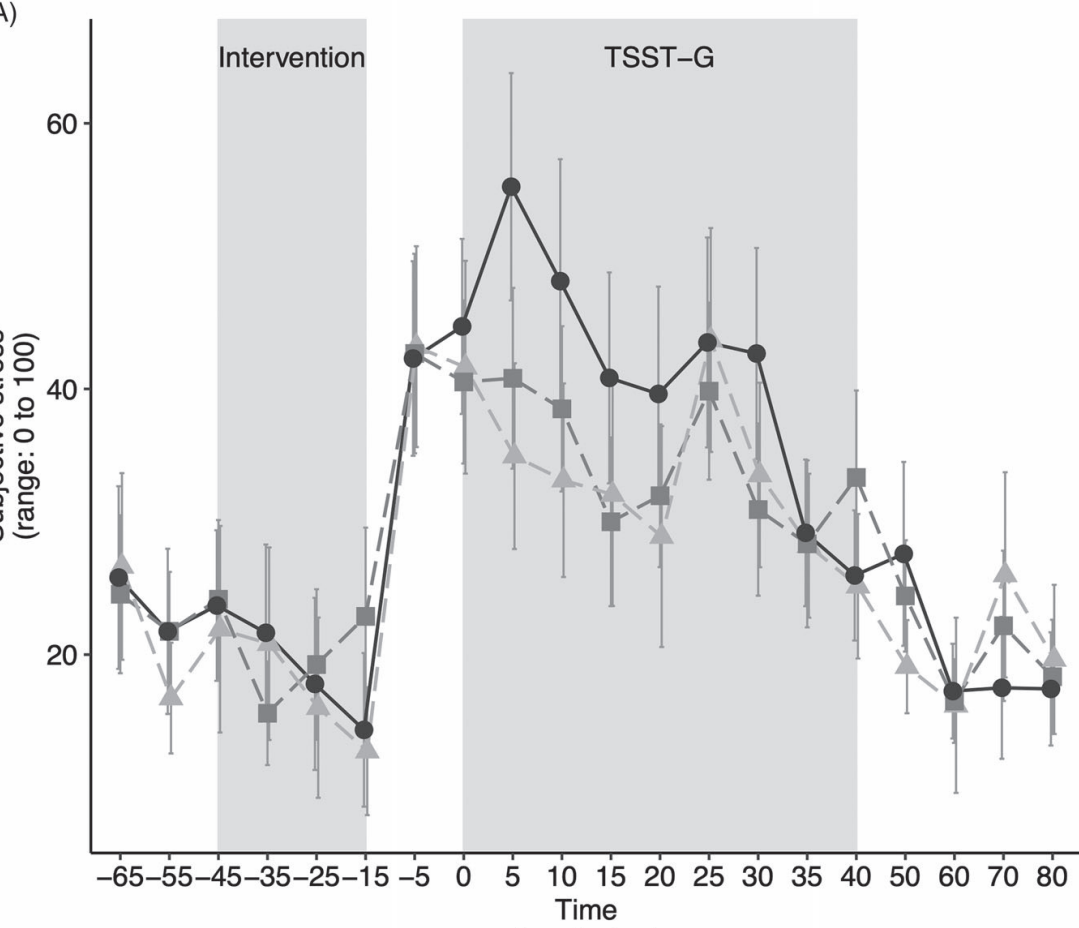

(in minutes)
(B)

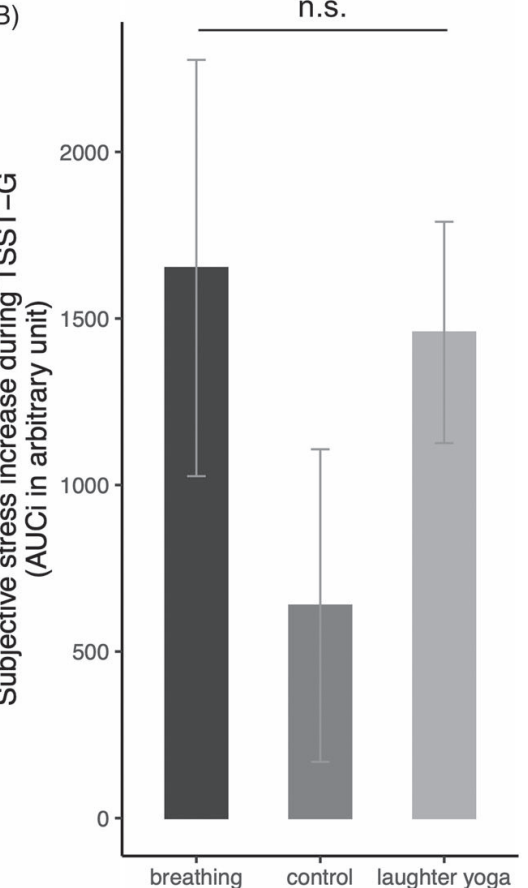

Experimental Group

Experimental Group - - Relaxation Breathing - - Control $-1-$ Laughter Yoga

Figure 4. (A) Subjective stress levels over the course of the experiment in the three experimental groups. (B) The subjective stress increase in response to the stressor, measured by the area under the curve with respect to the increase (AUCi), by experimental group. The subjective stress increase in response to the stressor did not differ significantly between the groups. Values are shown as mean \pm SD. TSST-G: modified version of the Trier Social Stress Test for Groups. 
or endocrine markers during the interventions itself, nor by differences in anxiety and depression. This effect was most pronounced looking at the cortisol response to the stressor, while the autonomic stress response as indicated by increases in alpha amylase was not significantly attenuated, although the effect on alpha amylase was descriptively mirroring the effects found in cortisol. Furthermore, subjective stress ratings did not differ among the groups; thus, it seems that all groups perceived the stressor as equally stressful on an emotional level. It is therefore unlikely that perceptual mechanisms are the driving forces for these results, unless the subjective stress response is not a good marker of individual perception. The changes in respiratory patterns during laughter (Ruch \& Ekman, 2001), and the relaxation following it, are improbable to account for this effect as well, as the relaxation breathing group with presumably similar increases in total lung volume, did not differ from the control group.

As remarked above, LY did not seem to influence the fast, autonomic stress response or whether a situation is perceived as stressful or not; instead, it seemed to specifically affect the response of the endocrine stress system. This was somewhat unexpected, and therefore warrants further discussion. The laboratory stress in this experiment was of social-evaluative nature, which is known to especially provoke a response in HPA axis activity (Dickerson \& Kemeny, 2004; Skoluda et al., 2015). Possible explanations for the attenuated cortisol stress response in the LY group are thus discussed in the light of the strengths and weaknesses of the current study.

One possible explanation for the observed effects may be the influence of social interaction and the consequential formation of a sense of belonging (Chang et al., 2013) and a shared social identity (Häusser et al., 2012) which might be enhanced in the LY group compared to both control groups. In contrast to participants in the relaxation breathing and the control intervention, which included only little, respectively no social interactions, participants in the LY group were actively encouraged to laugh with each other, and studies suggest that antiphonal laughter - laughter during or following another person's laugh - increases positive emotions toward that other person (Smoski \& Bachorowski, 2003). This interaction might have changed the extent to which participants were affected by the social threat they were confronted with during the stress task. Indeed, triggering a social identity in groups has been shown to buffer the endocrine stress response (Häusser et al., 2012). Moreover, instead of fearing to be negatively evaluated by both the committee, and the other group members, there might have been a shared feeling of empathy and social support within the study participants experiencing the TSST-G after they had a shared LY experience. A study by Heinrichs et al. (2003) showed that social support can lead to a lowered cortisol and subjective stress response following a stressor. Complementary to this argumentation, research suggests that the uncontrollability and social-evaluative threat elicited by a stressor is proportionally linked to the amount of secreted cortisol (Dickerson et al., 2004; Dickerson \& Kemeny, 2004). Despite a similar induction of distress and negative affect, stress protocols including social-evaluative threat (such as the TSST-G) are associated with greater cortisol increases compared to protocols without that component (such as the Cold Pressor Task; Skoluda et al., 2015). According to the Social Self Preservation Model, threats to the social self, such as situations in which there is a potential for negative evaluation of a core aspect of oneself, increase shame (Dickerson et al., 2004; Dickerson \& Kemeny, 2004). It has been proposed that shame might be the key affective component leading to the increase in cortisol (Dickerson et al., 2004). This assumption is supported by studies showing that participants with the greatest increases in shame also show the strongest cortisol response (Gruenewald et al., 2004). We hypothesize that the shared experience of laughter decreases the experience of shame during the TSST-G. When the stress protocol no longer poses a threat to the social self for the LY group, participants probably experience less shame which leads to an attenuated cortisol response compared to the relaxation breathing and control group. This effect might be mediated by a reduction in self-awareness and self-attention during laughter (Ruch \& Ekman, 2001), which would explain the specificity of the effect for LY. Taken together, the decreased cortisol stress response following the LY intervention could partially be explained by effects mediated by the social interaction that took place in the LY group. However, since participants in the relaxation breathing group also interacted with each other, yet did not show a similarly strong reduction in the cortisol stress response, we conclude the observed effects cannot be explained by social interaction alone.

Although LY was able to reduce the cortisol stress response, the subjective stress ratings were unaffected by the intervention. We assumed that LY would have the potential to influence both responses, however, the emotional and physiological stress response do not necessarily correspond (Ali et al., 2017; Campbell \& Ehlert, 2012). In accordance with the somatic marker hypothesis (Damasio et al., 1996), one could assume that the rating of a current emotional state relies on the self-percepted, acute physiological state one's body is currently in. Studies have shown that both, cardiovascular arousal and the experience of negative emotions are represented and processed in similar brain areas (Pollatos et al., 2007). Since the current state of the autonomic nervous system can be inferred through interoception focusing on the heart-rate, and the autonomic stress response was not as strongly affected by LY as the endocrine system, the subjective stress ratings might be a consequence of the activation of the sympathetic nervous system.

Summing up our findings, the data indicated that LY might present an effective intervention to reduce the cortisol response to acute stress. Although this response to acute stress is highly adaptive and supports the maintenance of homeostasis in the body, research also shows that prolonged or repeated activation of the HPA axis without habituation to the stressor is associated with psychopathology. In light of these findings, LY might prevent the accumulative adverse effects that stress may have on health. Nonetheless, it might not prevent from negative feelings that individuals are being exposed to during the stressful situation.

Taken together, this study was one of the first to investigate the effects of LY on the acute stress response in a controlled experimental setting. The strengths of this study are 
(1) the randomized assignment of participants to the experimental conditions, (2) the relaxation breathing control group that accounts for differences in oxygenation during laughter, (3) the standardized protocol used to induce stress, and (4) the assessment of both the physiological and psychological stress response throughout the experiment.

A number of limitations of the here reported findings should not go unmentioned, however. First, we controlled for the impact of breathing during laughter on the observed effects. Other mediating factors for the cortisol stress response include social interaction during the intervention, and menstrual cycle. Although the breathing groups comprised a social component, and therefore added some control for this factor, future studies should control for this confound more strictly. Second, we tried to control for possible effects of hormonal status of female participants through selfreported data. We realize that this method is unreliable, and would therefore suggest that this should ideally be observed objectively by means of LH tests in future studies. Third, the question remains why $L Y$ seemed to have an effect on cortisol levels but did not significantly change the autonomic stress response or how participants emotionally perceived the stressor. Remarkably, the effect of the interventions on alpha amylase was descriptively similar to the effects found in cortisol. Yet, the number of subjects in this study was rather smaller. Possibly, we could not detect an effect of LY on the autonomic stress response due to power constraints relating thereto. Studies with greater sample size would be desirable to replicate and extend the observed effects. Finally, more elaborate designs are necessary to determine what exactly made LY effective - the social aspects of the LY intervention, the laughter itself, or the bonding experience within the group that then went on to complete the TSST-G. Here, follow-up studies are needed to individually manipulate these various situational aspects and determine their effect, since the present study could not encounter for them. Further, longitudinal studies will have to determine whether these results translate into beneficial long-term adaptations that serve as a preventive and therapeutic option to prevent, attenuate and better understand stress-related disease.

Conclusively, although more research is still needed, LY might pose an attractive possibility to reduce the cortisol response to stress, thereby potentially attenuating stressrelated health problems, especially when considering the ease of implementation, and the cost-effectiveness.

\section{Disclosure statement}

No potential conflict of interest was reported by the author(s).

\section{Author contributions}

Maria Meier (MM), Lisa Wirz (LW) and Jens C. Pruessner (JCP) drafted the manuscript. MM, LW and JCP analyzed the data and interpreted the results. LW ran the overall study. Philip Dickinson (PD) conceived of the study research question to investigate the impact $L Y$ may have in mitigating acute stress. PD designed and ran the LY portion of the study PD, LW, JCP designed the integration of LY in the stress study, with input from JCP's lab. All authors read, revised and approved the final manuscript.

\section{Funding}

This work was supported by an operating grant of the Canadian Institutes of Health Research awarded to Jens C. Pruessner (MOP125913).

\section{Data availability statement}

The data that support the findings of this study are available from the corresponding author (MM) upon reasonable request.

\section{References}

Ali, N., Nitschke, J. P., Cooperman, C., \& Pruessner, J. C. (2017). Suppressing the endocrine and autonomic stress systems does not impact the emotional stress experience after psychosocial stress. Psychoneuroendocrinology, 78, 125-130. https://doi.org/10.1016/j.psyneuen.2017.01.015

Beck, A. T., Steer, R. A., \& Brown, G. K. (1996). Beck Depression InventoryII. The Psychological Corporation.

Beckman, H., Regier, N., \& Young, J. (2007). Effect of Workplace Laughter Groups on Personal Efficacy Beliefs. The Journal of Primary Prevention, 28(2), 167-182. https://doi.org/10.1007/s10935-007-0082-z

Bressington, D., Yu, C., Wong, W., Ng, T. C., \& Chien, W. T. (2018). The effects of group-based Laughter Yoga interventions on mental health in adults: A systematic review. Journal of Psychiatric and Mental Health Nursing, 25(8), 517-527. https://doi.org/10.1111/jpm.12491

Campbell, J., \& Ehlert, U. (2012). Acute psychosocial stress: Does the emotional stress response correspond with physiological responses? Psychoneuroendocrinology, 37(8), 1111-1134. https://doi.org/10.1016/j. psyneuen.2011.12.010

Chang, C., Tsai, G., \& Hsieh, C.-J. (2013). Psychological, immunological and physiological effects of a Laughing Qigong Program (LQP) on adolescents. Complementary Therapies in Medicine, 21(6), 660-668. https://doi.org/10.1016/j.ctim.2013.09.004

Cohen, J. (1973). Eta-squared and partial eta-squared in fixed factor anova designs. Educational and Psychological Measurement, 33(1), 107-112. https://doi.org/10.1177/001316447303300111

Cohen, J. (1988). Statistical power analysis for the behavioral sciences (2nd ed). L. Erlbaum Associates.

Creswell, J. D., Pacilio, L. E., Lindsay, E. K., \& Brown, K. W. (2014). Brief mindfulness meditation training alters psychological and neuroendocrine responses to social evaluative stress. Psychoneuroendocrinology, 44, 1-12. https://doi.org/10.1016/j.psyneuen.2014.02.007

Damasio, A. R., Everitt, B. J., \& Bishop, D. (1996). The somatic marker hypothesis and the possible functions of the prefrontal cortex [and discussion]. Philosophical Transactions: Biological Sciences, 351, 1413-1420.

Dickerson, S. S., Gruenewald, T. L., \& Kemeny, M. E. (2004). When the social self is threatened: Shame, physiology, and health. Journal of Personality, 72(6), 1191-1216. https://doi.org/10.1111/j.1467-6494.2004.00295.x

Dickerson, S. S., \& Kemeny, M. E. (2004). Acute stressors and cortisol responses: A theoretical integration and synthesis of laboratory research. Psychological Bulletin, 130(3), 355-391. https://doi.org/10. 1037/0033-2909.130.3.355

Dickinson, P., Looper, K. J., \& Groleau, D. (2011). Patients diagnosed with nonepileptic seizures: Their perspectives and experiences. Epilepsy \& Behavior : E\&B, 20(3), 454-461. https://doi.org/10.1016/j.yebeh.2010.12.034

Ditzen, B., Neumann, I. D., Bodenmann, G., von Dawans, B., Turner, R. A., Ehlert, U., \& Heinrichs, M. (2007). Effects of different kinds of couple interaction on cortisol and heart rate responses to stress in women. Psychoneuroendocrinology, 32(5), 565-574. https://doi.org/10.1016/j. psyneuen.2007.03.011

Farifteh, S., Mohammadi-Aria, A., Kiamanesh, A., \& Mofid, B. (2014). The impact of laughter yoga on the stress of cancer patients before chemotherapy. Iranian Journal of Cancer Prevention, 7(4), 179-183.

Fox, J., \& Weisberg, S. (2011). An $\{R\}$ companion to applied regression. 2nd ed. Sage. http://socserv.socsci.mcmaster.ca/jfox/Books/Companion 
Fox, J., \& Weisberg, S. (2019). An $\{R\}$ companion to applied regression. 3rd ed. Sage. http://tinyurl.com/carbook

Fujisawa, A., Ota, A., Matsunaga, M., Li, Y., Kakizaki, M., Naito, H., \& Yatsuya, H. (2018). Effect of laughter yoga on salivary cortisol and dehydroepiandrosterone among healthy university students: A randomized controlled trial. Complementary Therapies in Clinical Practice, 32, 6-11. https://doi.org/10.1016/j.ctcp.2018.04.005

Gruenewald, T. L., Kemeny, M. E., Aziz, N., \& Fahey, J. L. (2004). Acute threat to the social self: Shame, social self-esteem, and cortisol activity. Psychosomatic Medicine, 66(6), 915-924. https://doi.org/10.1097/01. psy.0000143639.61693.ef

Hammerfald, K., Eberle, C., Grau, M., Kinsperger, A., Zimmermann, A., Ehlert, U., \& Gaab, J. (2006). Persistent effects of cognitive-behaviora stress management on cortisol responses to acute stress in healthy subjects-a randomized controlled trial. Psychoneuroendocrinology, 31(3), 333-339. https://doi.org/10.1016/j.psyneuen.2005.08.007

Häusser, J. A., Kattenstroth, M., van Dick, R., \& Mojzisch, A. (2012). "We" are not stressed: Social identity in groups buffers neuroendocrine stress reactions. Journal of Experimental Social Psychology, 48(4), 973-977. https://doi.org/10.1016/j.jesp.2012.02.020

Heinrichs, M., Baumgartner, T., Kirschbaum, C., \& Ehlert, U. (2003). Socia support and oxytocin interact to suppress cortisol and subjective responses to psychosocial stress. Biological Psychiatry, 54(12), 1389-1398. https://doi.org/10.1016/S0006-3223(03)00465-7

Heo, E. H., Kim, S., Park, H.-J., \& Kil, S. Y. (2016). The effects of a simulated laughter programme on mood, cortisol levels, and health-related quality of life among haemodialysis patients. Complementary Therapies in Clinical Practice, 25, 1-7. https://doi.org/10.1016/j.ctcp.2016.07.001

Hothorn, T., Bretz, F., \& Westfall, P. (2008). Simultaneous inference in general parametric models. Biometrical Journal. Biometrische Zeitschrift, 50(3), 346-363. https://doi.org/10.1002/bimj.200810425

Kalia, M. (2002). Assessing the economic impact of stress-the modern day hidden epidemic. Metabolism: Clinical and Experimental, 51(6 Suppl 1), 49-53. https://doi.org/10.1053/meta.2002.33193

Kirschbaum, C., Klauer, T., Filipp, S.-H., \& Hellhammer, D. H. (1995). Sexspecific effects of social support on cortisol and subjective responses to acute psychological stress. Psychosomatic Medicine, 57(1), 23-31. https://doi.org/10.1097/00006842-199501000-00004

Kirschbaum, C., Pirke, K.-M., \& Hellhammer, D. H. (1993). The 'Trier Social Stress Test' - a tool for investigating psychobiological stress responses in a laboratory setting. Neuropsychobiology, 28(1-2), 76-81. https://doi. org/10.1159/000119004

Klaperski, S., von Dawans, B., Heinrichs, M., \& Fuchs, R. (2013). Does the level of physical exercise affect physiological and psychological responses to psychosocial stress in women? Psychology of Sport and Exercise, 14(2), 266-274. https://doi.org/10.1016/j.psychsport.2012.11. 003

Kuiper, N. A., \& Martin, R. A. (1998). Laughter and stress in daily life: relation to positive and negative affect. Motivation and Emotion, 22(2) 133-153. https://doi.org/10.1023/A:1021392305352

Lakens, D. (2013). Calculating and reporting effect sizes to facilitate cumulative science: A practical primer for t-tests and ANOVAs. Frontiers in Psychology, 4, 863. https://doi.org/10.3389/fpsyg.2013. 00863

Levine, T. R., \& Hullett, C. R. (2002). Eta squared, partial eta squared, and misreporting of effect size in communication research. Human Communication Research, 28(4), 612-625. https://doi.org/10.1111/j. 1468-2958.2002.tb00828.x

Martin, R. A. (2001). Humor, laughter, and physical health Methodological issues and research findings. Psychological Bulletin, 127(4), 504-519. https://doi.org/10.1037/0033-2909.127.4.504

Matousek, R. H., Pruessner, J. C., \& Dobkin, P. L. (2011). Changes in the cortisol awakening response (CAR) following participation in Mindfulness-Based Stress Reduction in women who completed treatment for breast cancer. Complementary Therapies in Clinical Practice, 17(2), 65-70. https://doi.org/10.1016/j.ctcp.2010.10.005

Miller, M., \& Fry, W. F. (2009). The effect of mirthful laughter on the human cardiovascular system. Medical Hypotheses, 73(5), 636-639. https://doi.org/10.1016/j.mehy.2009.02.044
Mora-Ripoll, R. (2011). Potential health benefits of simulated laughter: A narrative review of the literature and recommendations for future research. Complementary Therapies in Medicine, 19(3), 170-177. https:// doi.org/10.1016/j.ctim.2011.05.003

Polheber, J. P., \& Matchock, R. L. (2014). The presence of a dog attenuates cortisol and heart rate in the Trier Social Stress Test compared to human friends. Journal of Behavioral Medicine, 37(5), 860-867. https:// doi.org/10.1007/s10865-013-9546-1

Pollatos, O., Schandry, R., Auer, D. P., \& Kaufmann, C. (2007). Brain structures mediating cardiovascular arousal and interoceptive awareness. Brain Research, 1141, 178-187. https://doi.org/10.1016/j.brainres.2007. 01.026

Provine, R. R. (1992). Contagious laughter: Laughter is a sufficient stimulus for laughs and smiles. Bulletin of the Psychonomic Society, 30(1), 1-4. https://doi.org/10.3758/BF03330380

Proyer, R. T., Ruch, W., \& Rodden, F. A. (2012). Letter on Shahidi et al. (2011): "Laughter Yoga versus group exercise program in elderly depressed women: A randomized controlled trial" I - First things first! Caveats in research on "Laughter yoga". International Journal of Geriatric Psychiatry, 27(8), 873-874. https://doi.org/10.1002/gps.2793

Pruessner, J. C., Kirschbaum, C., Meinlschmid, G., \& Hellhammer, D. H. (2003). Two formulas for computation of the area under the curve represent measures of total hormone concentration versus timedependent change. Psychoneuroendocrinology, 28(7), 916-931. https:// doi.org/10.1016/S0306-4530(02)00108-7

$\mathrm{R}$ Core Team (2019). R: A language and environment for statistical computing. R Foundation for Statistical Computing. https://www.R-project. org/

RStudio Team (2016). RStudio: Integrated development for $R$ [Computer Software]. URL http://www.rstudio.com/. Boston, MA: RStudio, Inc.

Ruch, W., \& Ekman, P. (2001). The expressive pattern of laughter. In A. Kaszniak (Ed.), Emotions, qualia, and consciousness (pp. 426-443). World Scientific Publisher. https://doi.org/10.1142/9789812810687_ 0033

Savage, B. M., Lujan, H. L., Thipparthi, R. R., \& DiCarlo, S. E. (2017). Humor, laughter, learning, and health! A brief review. Advances in Physiology Education, 41(3), 341-347. https://doi.org/10.1152/advan. 00030.2017

Skoluda, N., Strahler, J., Schlotz, W., Niederberger, L., Marques, S., Fischer, S., Thoma, M. V., Spoerri, C., Ehlert, U., \& Nater, U. M. (2015). Intra-individual psychological and physiological responses to acute laboratory stressors of different intensity. Psychoneuroendocrinology, 51, 227-236. https://doi.org/10.1016/j.psyneuen.2014.10.002

Smoski, M., \& Bachorowski, J.-A. (2003). Antiphonal laughter between friends and strangers. Cognition \& Emotion, 17(2), 327-340. https://doi. org/10.1080/02699930302296

Spielberger, C. D., Sydeman, S. J., Owen, A. E., \& Marsh, B. J. (1999). Measuring anxiety and anger with the State-Trait Anxiety Inventory (STAI) and the State-Trait Anger Expression Inventory (STAXI). Lawrence Erlbaum Associates Publishers.

Tanaka, A., Tokuda, N., \& Ichihara, K. (2018). Psychological and physiological effects of laughter yoga sessions in Japan: A pilot study. Nursing \& Health Sciences, 20(3), 304-312. https://doi.org/10.1111/nhs. 12562

von Dawans, B., Kirschbaum, C., \& Heinrichs, M. (2011). The Trier Social Stress Test for Groups (TSST-G): A new research tool for controlled simultaneous social stress exposure in a group format. Psychoneuroendocrinology, 36(4), 514-522. https://doi.org/10.1016/j. psyneuen.2010.08.004

Weinberg, M. K., Hammond, T. G., \& Cummins, R. A. (2013). The impact of laughter yoga on subjective wellbeing: A pilot study. European Journal of Humour Research, 1(4), 25-34. https://doi.org/10.7592/EJHR2013.1.4. weinberg

Wickham, H. (2016). ggplot2: Elegant graphics for data analysis. SpringerVerlag.

Yim, J. (2016). Therapeutic benefits of laughter in mental health: A theoretical review. The Tohoku Journal of Experimental Medicine, 239(3), 243-249. https://doi.org/10.1620/tjem.239.243 